\title{
Laser ablation synthesis of monodispersed magnetic alloy nanoparticles
}

\author{
Takafumi Seto ${ }^{1,2, *}$, Kenji Koga ${ }^{1,3}$, Hiroyuki Akinaga ${ }^{1,3}$, Fumiyoshi Takano ${ }^{1,3}$, Takaaki Orii ${ }^{1,2}$ and \\ Makoto Hirasawa ${ }^{1,2}$ \\ ${ }^{1}$ Research Consortium for Synthetic Nano-Function Materials Project (SYNAF), National Institute of \\ Advanced Industrial Science and Technology (AIST), Central 2, 1-1-1 Umezono, Tsukuba, Ibaraki, 305- \\ 8568, Japan; ${ }^{2}$ Advanced Manufacturing Research Institute, National Institute of Advanced Industrial Science \\ and Technology (AIST), 1-2-1 Namiki, Tsukuba, 305-8564, Japan; ${ }^{3}$ Nanotechnology Research Institute, \\ National Institute of Advanced Industrial Science and Technology (AIST), AIST Central 5, 1-1-1 Higashi, \\ Tsukuba, 305-8565, Japan; *Author for correspondence (Tel.: +81-29-861-7268; E-mail: t.seto@aist.go.jp)
}

Received 1 July 2005; accepted in revised form 5 July 2005

Key words: aerosol, nanoparticle, laser ablation, alloy, magnetic property, classification

\begin{abstract}
Monodispersed CoPt alloy nanoparticles were synthesized by a pulsed laser ablation (PLA) technique coupled with a low-pressure operating differential mobility analyzer (LP-DMA). The CoPt alloy nanoparticles were generated by laser ablating a solid $\mathrm{Co}-\mathrm{Pt}$ target. In CoPt alloy nanoparticles synthesized from a target with a Co composition of 75 at \%, the nanoparticle surfaces were covered by an oxide layer and exhibited a core-shell structure. In contrast, no shell was observed in particles generated from a target with a Co:Pt ratio of 50:50 at\%. According to an EDX analysis, the compositions of the individual nanoparticles were almost the same as that of the target material. Finally, the magnetic hysteresis loops of the CoPt alloy nanoparticles exhibited ferromagnetism.
\end{abstract}

\section{Introduction}

With the recent progress in nanotechnology, manufacturers face a growing need for fabrication processes with nanometer-scaled precision and high production yields. Due to the drastic change in the electron structures, nanoparticles, particularly smaller than $20 \mathrm{~mm}$ in diameter, exhibit unique properties unseen in bulk materials. For this reason, nanoparticles are now considered as promising building blocks for nano-structured materials and nano-devices. Nanoparticles are conventionally prepared via a liquid phase process that provides a low cost and permits good control over the particle sizes and shapes. In spite of these advantages, however, the solvents and precursors in the liquid phase process damage the generated nanoparticles by introducing a significant level of impurity into the system. To reduce the impurities, the liquid phase process is followed up with annealing or other after-treatments that agglomerate the nanoparticles.

Laser ablation, an alternative to the liquid phase process, adopts a novel gas phase method. When a solid target material is irradiated by a high-energy laser beam, the material vaporizes and condenses into nanoparticles. Compared with dry processes such as physical and chemical vapor condensation, laser ablation is a typical 'cold wall' process wherein the laser beam heats up a specific part of the target. 
The precise focusing capability of the laser provides several advantages: in addition to enabling almost complete elimination of impurities, it provides a high energy density (gigawatt level) capable of vaporizing high-melting-point materials, including ceramics.

As the nanoparticles generated by laser ablation are free from contaminants, they exhibit various nano-scale properties. The chain agglomerates of metal oxides generated by laser ablation show elastic properties under the electron microscope (Ogawa et al., 2000). Laser-synthesized silicon nanoparticles emit visible light at room temperature (Yamada et al., 1996; Orii et al., 2003). El-Shall (1996) developed a method of laser-vaporization-controlled condensation (LVCC) and used it to fabricate various kinds of nanoparticles. The chief drawback of the laser ablation approach is the difficulty in controlling the nanoparticle properties due to the relatively wide size distribution and agglomeration. Several groups have investigated the size distribution of laser-ablation-generated nanoparticles using a low pressure operating differential mobility analyzer (LP-DMA) technique (Kawakami et al., 1999; Makino et al., 1999; Camata et al., 2000). According to their observations, the agglomeration can be minimized by reducing the operating pressure. LP-DMA can be operated at a pressure as low as $600 \mathrm{~Pa}$ (Seto et al., 2001a, b), Suzuki et al. (2001) a level that makes it compatible with the laser ablation process under optimum conditions. This combination of laser ablation and LP-DMA provides a powerful tool for the analysis of various properties of nanoparticles. In the last 2 years, for example, we have used this combination to study the size-dependent optical properties of Si nanoparticles (Orii et al., 2003) and the magnetic properties of $\mathrm{Ni}$ nanoparticles (Seto et al., 2004).

In the present study we make a first attempt to synthesize metallic nanoparticles of $\mathrm{CoPt}$ alloy by laser ablation. Because of their large magnetic anisotropy, CoPt and FePt alloy nanoparticles are thought to hold great promise as candidates for the next generation of ultrahigh-density magnetic recording media (Sun et al., 2000; Yu et al., 2002; Sui et al., 2003). When these alloys are synthesized by the conventional methods of liquid phase process and sputtering, however, the size and crystal structure cannot be controlled with the precision required to obtain large magnetic anisotropy. In the following section we describe our attempts to synthesize monodispersed CoPt alloy nanoparticles.

\section{Laser synthesis of monodispersed nanoparticles}

As in other gas phase processes, the size distribution of nanoparticles generated by laser ablation is rather wide due to the random Brownian collision between primary particles. In the hopes of finding a solution, we are now developing a method to obtain nanometer-scale prototype devices using monodispersed nanoparticles as building blocks by combining aerosol techniques with laser ablation. As shown in the schematic illustration in Figure 1, the particles generated by laser ablation are transported by the gas stream and then sintered into a single sphere by aerosol post-annealing. The process significantly improves both the shape and crystallinity. After annealing, the polydispersed aerosols are classified by the balance between the electrostatic mobility and gas velocity using LPDMA. Finally, monodispersed particles are deposited onto the substrate.

This system has four important advantages.

(a) The composition of the nanoparticles is almost the same as that of the target material since the nanoparticles are formed from the re-condensed atoms of the vaporized target material.

(b) The crystal structure can be controlled by performing the post-annealing separately from the generation and classification.

(c) The LP-DMA precisely controls the size of the nanoparticles with a geometric standard deviation of less than 1.2.

(d) The entire process is sequential and nanoparticles can be synthesized without exposure to room atmosphere.

In order to synthesize alloy nanoparticles, we used a sintered Co-Pt powder mixture as a target. Once the nanoparticles were obtained, we analyzed them by a high-resolution transmission electron microscope (HR-TEM), electron diffraction (ED), and an energy dispersive X-ray (EDX) spectrometer.

\section{Experimental procedures}

The details of the experimental system are described elsewhere (Seto et al., 2004). A double 


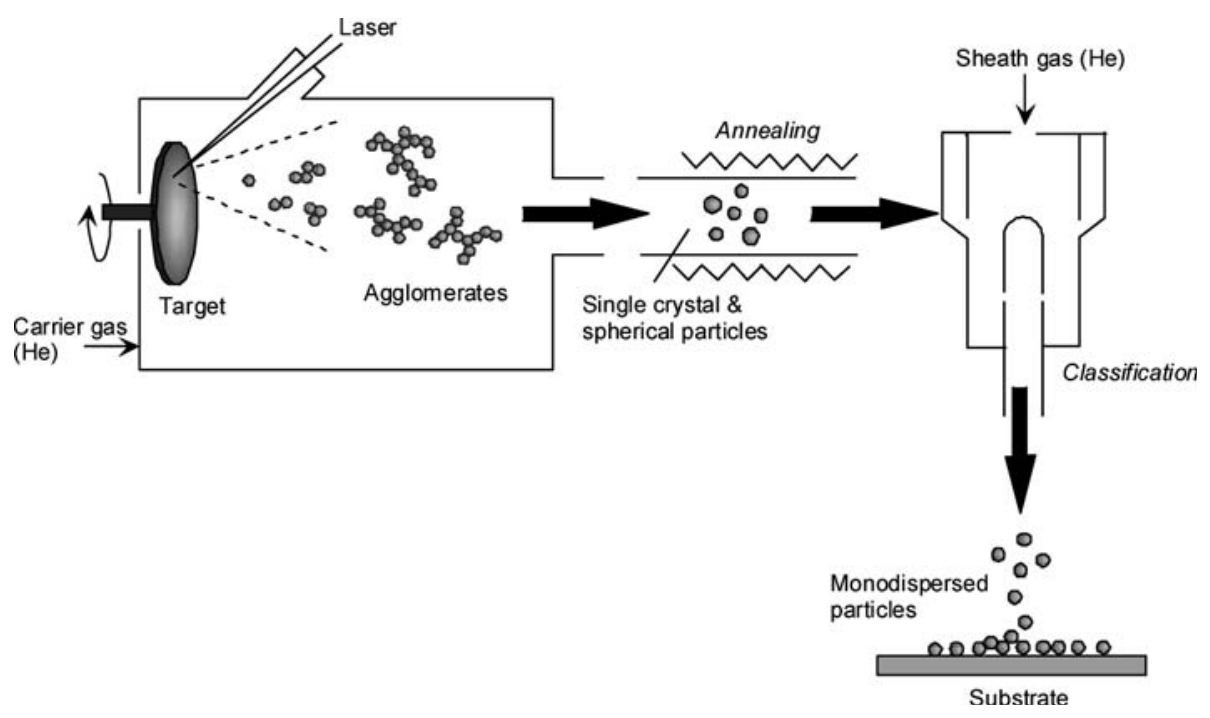

Figure 1. Laser synthesis of monodispersed nanoparticles.

harmonic wave of a pulsed Nd:YAG laser (model INDI, Spectra Physics Inc, wavelength $532 \mathrm{~nm}$, pulse width about $5 \mathrm{~ns}$, spot diameter $2 \mathrm{~mm}$, pulse energy $160 \mathrm{~mJ} /$ pulse, frequency $20 \mathrm{~Hz}$ ) was used for the ablation. High purity helium $(99.999 \%)$ was used as carrier gas and sheath gas of LPDMA). The flow rates of carrier gas and sheath gas were 0.4 and 2.0 standard liter per minute, respectively. The pressure of the system was controlled to be $1000 \mathrm{~Pa}$ by the valves. As shown in Figure 1, the laser beam was focused to a diameter of $2 \mathrm{~mm}$ on a rotating target disk $(50 \mathrm{~mm}$ dia., rotating speed $20 \mathrm{rpm}$ ). Targets with two different compositions were used to generate the $\mathrm{CoPt}$ alloy nanoparticles ( $\mathrm{Co}: \mathrm{Pt}=50: 50$ and 75:25 at \%). The ablated species, that is, the atoms and clusters, formed into laser-induced plasma (see Figure 2). The nanoparticles were produced by the condensation of ablated species at the edge of the plasma region. In the aerosol post-annealing, the particles were transported by the carrier gas with the flow rate of 0.4 standard liter per minute and then introduced into the tubular furnace maintained at a temperature of $1000^{\circ} \mathrm{C}$. An alumina tube with $12 \mathrm{~mm}$ in inner diameter and $600 \mathrm{~mm}$ in length was used as an annealing tube. The effective heating zone of the furnace was $200 \mathrm{~mm}$. Thus, the total residence time from ablation chamber to LPDMA and the residence time in the heating zone were $66 \mathrm{~ms}$ and $6 \mathrm{~ms}$, respectively. The residence time in the heating zone was relatively short, but this was enough to sinter the particles into single spheres, as shown later.

The LP-DMA was a modified version of the Vienna type DMA operable under reduced pressures as low as $200 \mathrm{~Pa}$. The performance of the LPDMA used in this study was calibrated in our previous report (Kim et al., 2004). We used deposition nozzle with $2 \mathrm{~mm}$ in diameter and the Stokes number is larger than unity for the particles with $5 \mathrm{~nm}$ in diameter. Thus, the particles were deposited by the inertial impaction. After the size-classification by the DMA, the particles were deposited onto the substrate (TEM micro-grid for HR-TEM, ED and EDX; Si for SQUID magnetometry).

\section{Results}

\section{Morphology of the generated particles}

Figure 3 shows the size distribution of CoPt alloy nanoparticles measured by a LP-DMA and an electrometer. As shown in Figure 3, the average diameter decreased from $19 \mathrm{~nm}$ to $14 \mathrm{~nm}$ with increasing annealing temperature due to the sintering of agglomerates.

Figure 4 shows the transmission electron micrographs of CoPt alloy nanoparticles generated from the two target compositions. The 


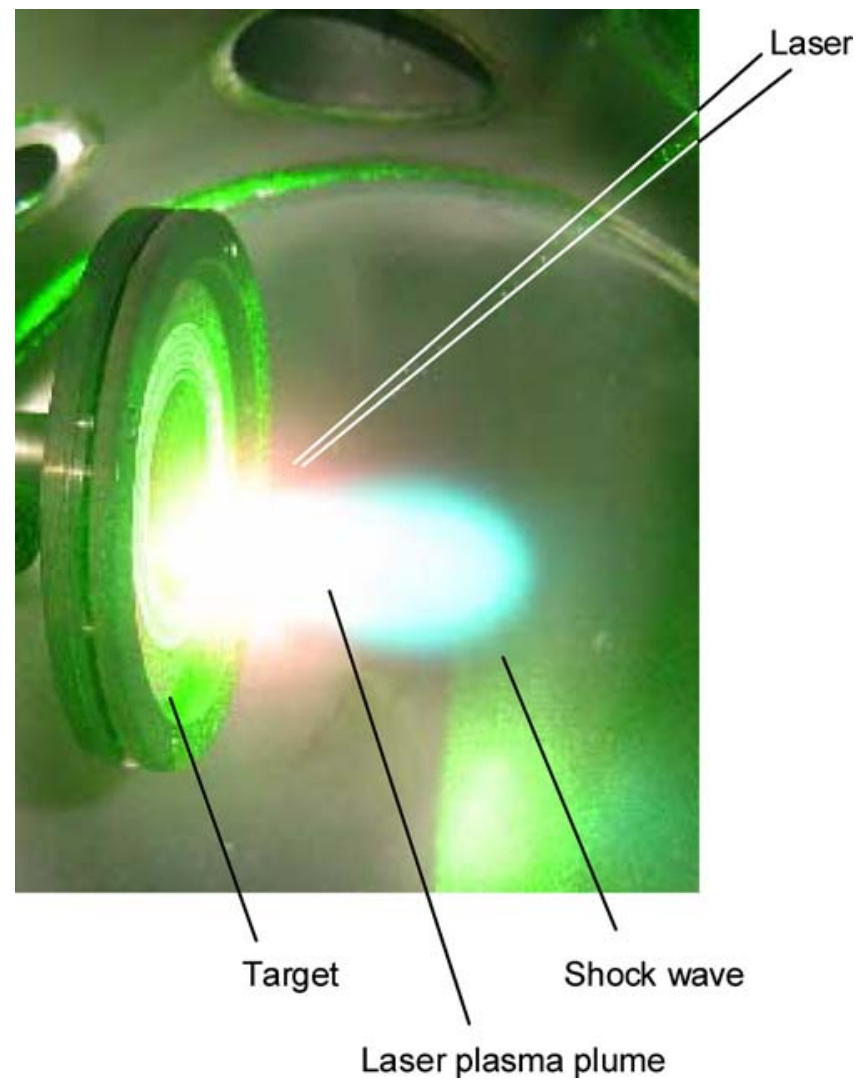

Figure 2. Laser induced plasma plume.

particles were classified by DMA to be $20 \mathrm{~nm}$ in mobility equivalent diameter by LP-DMA. In both compositions, the particles were spherical and single crystalline due to the aerosol postannealing process at $1000^{\circ} \mathrm{C}$. As shown in Figure $4 \mathrm{a}$, the shell covered surfaces of the particles generated from the $75: 25$ at $\%$ target, resulting in core-shell structures. The shell is considered to be cobalt oxide generated by the reaction between oxygen and the excess Co atoms at surface during exposure to room air. Similar core-shell structures have also been observed for Nickel particles (Sakiyama et al., 2004). On the other hand, the particles generated from the 50:50 at $\%$ target completely lacked a surface oxide layer. From the phase diagram of the Co-Pt system, the one-toone alloy of CoPt was dominant from about 40 to $75 \%-\mathrm{Pt}$ at room temperature. Given that the $\mathrm{CoPt}$ alloy should be anti-oxidative if formed, the excess Cobalt atoms in the composition (about $15 \%$ in the case of $75: 25 \%$ target) are separated out from alloy and they were thought to contribute to the oxide layer formation. On this basis, we speculate that core-shell structures can be realized when the Co content is more than $60 \%$ and that the thickness of the shell can be controlled by changing the composition of the target material.

In observing the electron diffraction (Figure 5a and $b$ ), we see that the patterns coincided with those from the CoPt alloy indicated for each plane. Further, the diffraction patterns of the two nanoparticle compositions were completely the same. Given the absence of patterns such as those observed in the $\mathrm{CoO}$ shells shown in Figure 5a, it appeared that the shells were amorphous. Typical patterns from the ordered structure $\left(L 1_{0}, \mathrm{CuAu}\right.$ structure) such as 100 and 110 were not observed from either of the nanoparticle compositions. Thus, we concluded that the annealing in the gas phase was sufficient to sinter the particles into single spheres and a single crystalline form, but not 


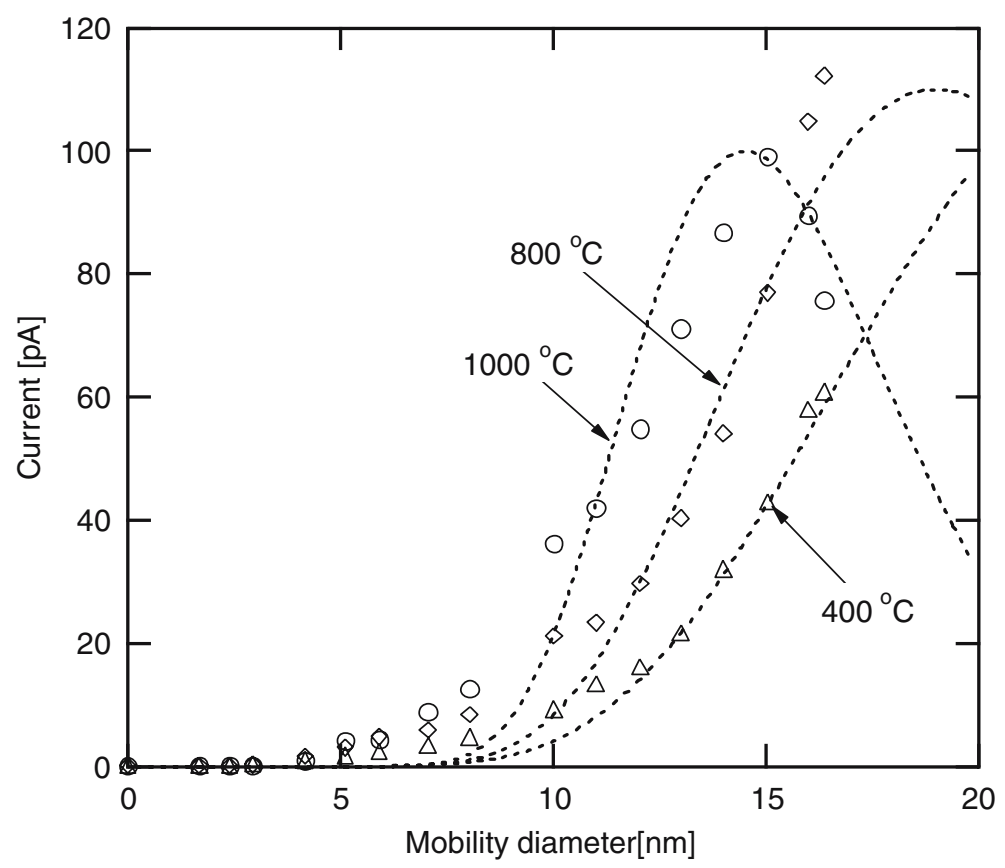

Figure 3. Size distribution of CoPt alloy nanoparticles for the different annealing temperatures.
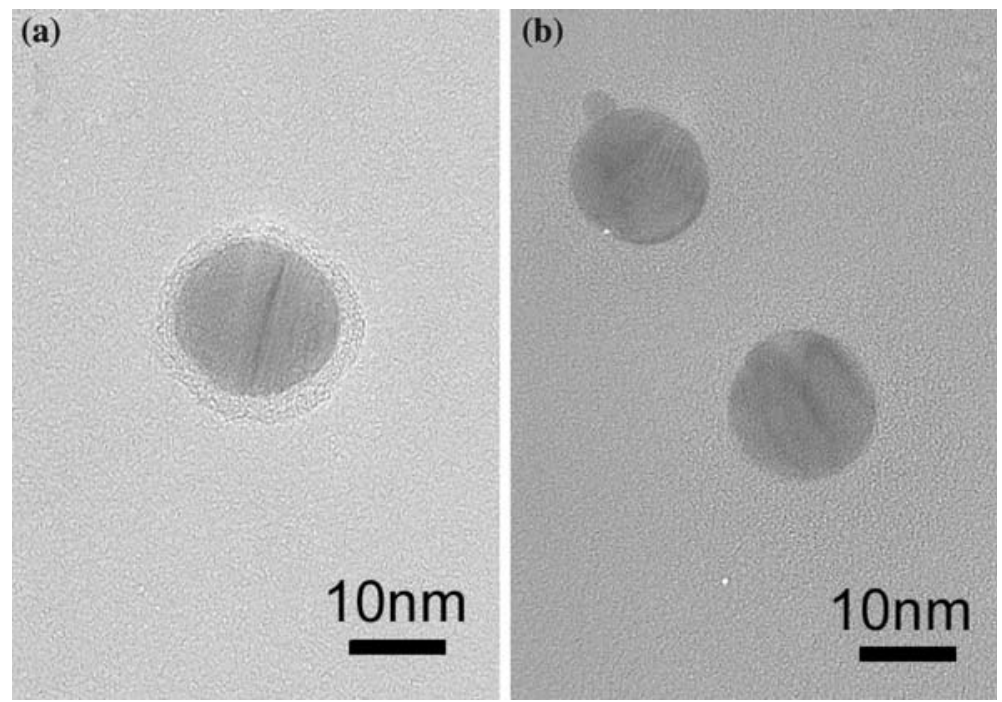

Figure 4. Transmission electron micrographs of $\mathrm{CoPt}$ alloy nanoparticles. (a) CoPt alloy nanoparticle generated by laser ablation of Co-Pt target with Co:Pt composition of 75:25 at\%, and (b) those generated from 50:50 at \% target.

to order the atomic structure. Further annealing after the deposition (typically more than $30 \mathrm{~min}$, Sun et al., 2000) is required to obtain ordered $L 1_{0}$ phase.
Figure 6 shows a typical energy dispersive X-ray (EDX) spectrum from single 20-nm CoPt alloy nanoparticles generated from the 50:50 at $\%$ target. The EDX apparatus was attached to the 


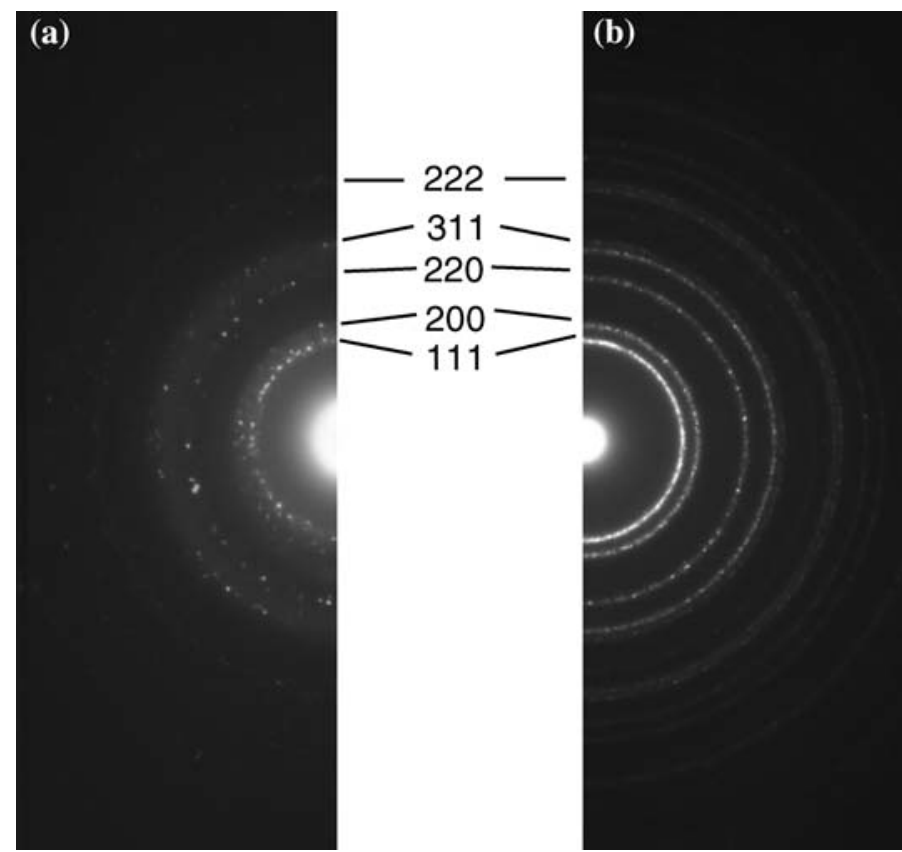

Figure 5. Electron diffraction patters of CoPt alloy nanoparticles. (a) CoPt alloy nanoparticle generated by laser ablation of Co-Pt target with Co:Pt composition of 75:25 at\%, and (b) those generated from 50:50 at \% target.

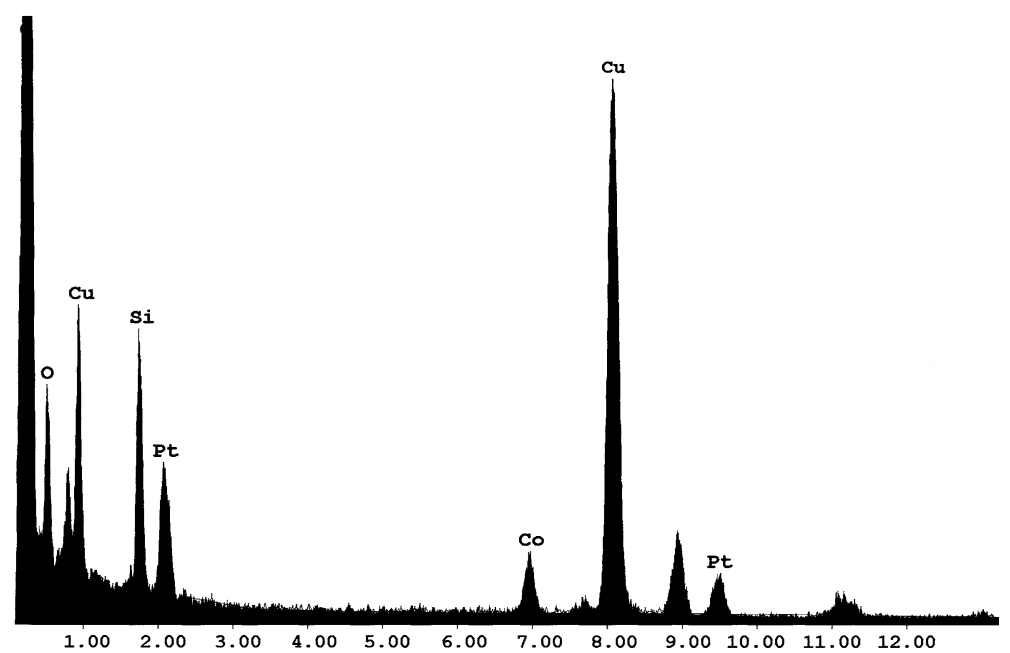

Figure 6. EDX spectrum from single CoPt alloy nanoparticle $(20 \mathrm{~nm})$ generated by the laser ablation of $\mathrm{Co}: \mathrm{Pt}=50: 50$ at $\%$ target.

HR-TEM and operated to obtain a signal from each nanoparticle focused in the HR-TEM view scope (e.g., Figure 4a). The peaks of $\mathrm{Co}$ and $\mathrm{Pt}$ from the spectrum were clearly distinguishable from some of the background peaks of silicon and copper. An element analysis from Co-K and Pt-L bands provided us with the composition data shown in Table 1. Based on this data, we found that the generated nanoparticles had almost same (slightly lower Co) composition as the target. The 
Table 1. EDX element analysis data for 14 individual nanoparticles

\begin{tabular}{clll}
\hline No. & $d_{\mathrm{p}}(\mathrm{nm})$ & Co at $\%$ & Pt at $\%$ \\
\hline 1 & 15 & 43.9 & 56.1 \\
2 & 15 & 43.9 & 56.1 \\
3 & 15 & 40.4 & 59.6 \\
4 & 15 & 44.9 & 55.1 \\
5 & 15 & 44.6 & 55.4 \\
6 & 15 & 44.5 & 55.5 \\
7 & 15 & 42.9 & 57.1 \\
Ave. & & $43.6 \pm 3.2$ & $56.4 \pm 3.2$ \\
8 & 20 & 49.3 & 50.7 \\
9 & 20 & 48.7 & 51.3 \\
10 & 20 & 44.1 & 55.9 \\
11 & 20 & 45.3 & 54.7 \\
12 & 20 & 45.1 & 54.9 \\
13 & 20 & 44.1 & 55.9 \\
14 & 20 & 47.7 & 52.3 \\
Ave. & & $46.3 \pm 3$ & $53.7 \pm 3$ \\
\hline
\end{tabular}

discrepancy, we concluded, was probably due to the different condensation rates of $\mathrm{Pt}$ and $\mathrm{Co}$ during particle generation. The Co composition of the particles of at least $20 \mathrm{~nm}$ in mobility diameter was about $3 \%$ larger than that of the particles with a diameter of $15 \mathrm{~nm}$ or less. It should be noted that the deviation of the composition was small (about 3\%) in both diameters.

\section{Magnetic property of CoPt alloy nanoparticles}

The ordered $L 1_{0}$ structure of CoPt is known to have a high magnetic anisotropy suitable for use in nano-magnets or high-density magnetic recording media. In the present experiment we measured the magnetic properties of laser-synthesized nanoparticles using a superconducting quantum interference device (SQUID) magnetometer. In brief, nanoparticles of $15-\mathrm{nm}$ in mobility diameter were deposited on a silicon substrate and then annealed at $700^{\circ} \mathrm{C}$ for $2 \mathrm{~h}$ to transfer the crystal structure from random face-centered-cubic (fcc) to ordered face-centered-tetragonal. As shown in Figure 7, ferromagnetism was observed at $5 \mathrm{~K}$. The magnetization was about $83.7 \mathrm{emu} / \mathrm{g}$, almost double the magnetization of bulk CoPt $(44 \mathrm{emu} / \mathrm{g})$, when bulk density $(8.12 \mathrm{~g} / \mathrm{cc})$ was applied for the calculation. Similar results have been obtained with measurements for Ni (Seto et al., 2004). On the other hand, the coercive force was smaller than the reported value for ordered $L 1_{0}$ composed of chemically synthesized CoPt alloy nanoparticles (24.2 kOe; Sui et al., 2003). This was probably due to the lack of ordering or to the slight difference in the composition.

\section{Summary}

CoPt alloy nanoparticles were synthesized by laser ablation coupled with the LP-DMA technique. The shape and crystallinity were both improved by the aerosol post-annealing process. The composition of the generated nanoparticles was almost identical to that of the target material. The generated

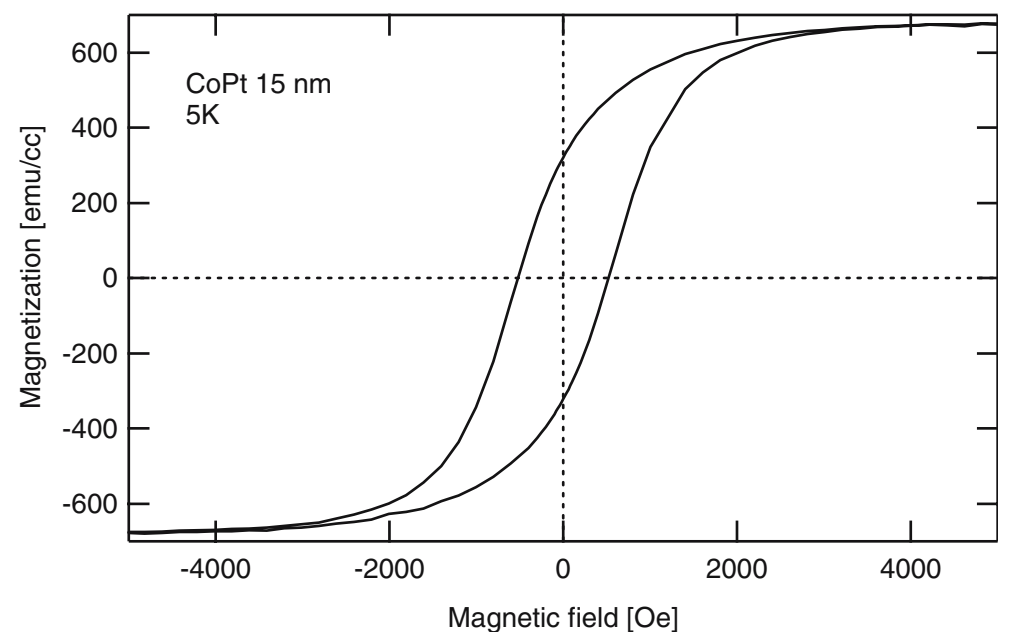

Figure 7. Magnetic hysteresis curve for the CoPt alloy nanoparticles generated by laser ablation. The particles were classified to be $15 \mathrm{~nm}$ by LP-DMA and annealed at $700^{\circ} \mathrm{C}$ for $2 \mathrm{~h}$. 
nanoparticles exhibited ferromagnetism at $5 \mathrm{~K}$. We demonstrated that the current process has a significant potential to analyze various properties of nanoparticles for other material systems.

\section{Acknowledgements}

This study was supported in part by the New Energy and Industrial Technology Development Organization (NEDO) under the Nanotechnology Materials Program.

\section{References}

Camata R.P., M. Hirasawa, K. Okuyama \& K. Takeuchi, 2000. Observation of aerosol formation during laser ablation using a low-pressure differential mobility analyzer. J. Aerosol Sci. 31, 391-401.

El-Shall M.S., 1996. Laser vaporization for the synthesis of nanoparticles and polymers containing metal particulates. Appl. Surf. Sci. 106, 347-355.

Kawakami Y., T. Seto \& E. Ozawa, 1999. Characterisitics of tungsten ultra-fine particles produced by $\mathrm{Nd}$ :YAG laser irradiation. Appl. Phys. A 69-S, 249-252.

Kim J., T. Seto, K. Sakiyama \& D. Kim, 2004. Characterization of low pressure DMA system for the size selection of magnetic nano-particles. Appl. Phys. A 79, 1497-1499.

Makino T., N. Suzuki, Y. Yamada, T. Yoshida, T. Seto \& N. Aya, 1999. Size classification of Si nanoparticles formed by pulsed laser ablation in helium background gas. Appl. Phys. A 69-S, 243-247.

Ogawa K., T. Vogt, M. Ulmann, S. Johnson \& S.K. Friedlander, 2000. Elastic properties of nanoparticle chain aggregates of
$\mathrm{TiO}_{2}, \mathrm{Al}_{2} \mathrm{O}_{3}$, and $\mathrm{Fe}_{2} \mathrm{O}_{3}$ generated by laser ablation. J. Appl. Phys. 87, 63-73.

Orii T., M. Hirasawa \& T. Seto, 2003. Tunable narrow-band light emission from size-selected $\mathrm{Si}$ nanoparticles produced by pulsed laser ablation. Appl. Phys. Lett. 83, 3395-3397.

Sakiyama K., K. Koga, T. Seto, M. Hirasawa \& T. Orii, 2004. Formation of size-selected $\mathrm{Ni} / \mathrm{NiO}$ core-shell particles by pulsed laser ablation. J. Phys. Chem. B 108, 523-529.

Seto T., Y. Kawakami, N. Suzuki, M. Hirasawa \& N. Aya, 2001a. Laser synthesis of uniform silicon single nanodots. Nano Letters 1, 315-318.

Seto T., Y. Kawakami, N. Suzuki, M. Hirasawa, S. Kano, N. Aya, S. Sasaki \& H. Shimura, 2001b. Evaluation of morphology and size distribution of silicon and titanium oxide nanoparticles generated by laser ablation. J. Nanoparticle Res. 3, 185-191.

Seto T, K. Koga, H. Akinaga, F. Takano, K. Sakiyama, M. Hirasawa \& T. Orii, 2004. Laser synthesis and magnetic properties of monodispersed core-shell nanoparticles. Appl. Phys. A 79, 1165-1167.

Sui Y., L. Yue, R. Skomski, X.Z. Li, J. Zhou \& D.J. Sellmyer, 2003. CoPt hard magnetic nanoparticle films synthesized by high temperature chemical reduction. J. Appl. Phys. 93, 7571-7573.

Sun S.H., C.B. Murray, D. Weller, L. Folks \& A. Moser, 2000. Monodisperse FePt nanoparticles and ferromagnetic FePt nanocrystal superlattices. Science 287, 1989.

Suzuki N., T. Makino, Y. Yamada, T. Yoshida \& T. Seto, 2001. Monodispersed, nonagglomerated silicon nanocrystallites. Appl. Phys. Lett. 78, 2043-2045.

Yamada Y., T. Orii, I. Umezu, S. Takeyama \& T. Yoshida, 1996. Optical properties of silicon nanocrystallites prepared by excimer laser ablation in inert gas. Jpn. J. Appl. Phys. 35, 1361-1365.

Yu A.C.C., M. Mizuno, Y. Sasaki, H. Kondo \& K. Hiraga, 2002. Structural characteristics and magnetic properties of chemically synthesized CoPt nanoparticles. Appl. Phys. Lett. 81, 3768-3770. 